\title{
Tumor Microenvironment-Responsive BSA Nanocarriers For Combined Chemo/Chemodynamic Cancer Therapy
}

\section{Ruiyi Zhang}

Anhui Polytechnic University

Teng Liu ( $\square$ tliu13@suda.edu.cn )

Soochow University https://orcid.org/0000-0002-2185-7260

\section{Wanzhen Li}

Anhui Polytechnic University

\section{Zhiyuan Ma}

Anhui Polytechnic University

Pei Pei

Soochow University

Weiwei Zhang

Anhui Polytechnic University

Kai Yang

Soochow University

Yugui Tao

Anhui Polytechnic University

\section{Research Article}

Keywords: Tumor microenvironment, chemodynamic therapy, chemotherapy, bovine serum albumin, nanoscale coordination polymers

Posted Date: February 10th, 2022

DOI: https://doi.org/10.21203/rs.3.rs-1331161/v1

License: (c) (i) This work is licensed under a Creative Commons Attribution 4.0 International License. Read Full License 


\section{Abstract}

Tumor microenvironment (TME), characterized by high glutathione (GSH), high hydrogen peroxide $\left(\mathrm{H}_{2} \mathrm{O}_{2}\right)$ and low acidic levels, is favorable for the growth, invasion and metastasis of cancer cells. Taking advantage of the specific characteristics of tumors, TME-responsive GCBD NPs are designed to deliver nanoscale coordination polymers (NCPs, GA-Cu) and chemotherapy drugs (doxorubicin, DOX) based on bovine serum albumin (BSA) nanocarriers into cancer cells for combined chemodynamic therapy (CDT) and chemotherapy. In an acidic environment, GCBD NPs could release approximately $90 \%$ copper ions, which can not only consume overexpressed GSH to modulate the TME but can also react with endogenous $\mathrm{H}_{2} \mathrm{O}_{2}$ in a Fenton-like reaction to achieve the CDT effect. Meanwhile, the released DOX could enter the nucleus of tumor cells and affect their proliferation to achieve efficient chemotherapy. Both in vitro and in vivo experiments showed that GCBD NPs had good biosafety and could effectively inhibit the growth of cancer cells. GCBD NPs are promising as a biocompatible nanoplatform to exploit TME characteristics for combined chemo and chemodynamic therapy, providing a novel strategy to eradicate tumors with high efficiency and specificity.

\section{Introduction}

The tumor microenvironment (TME) is an acidic environment that contains various cell types (such as tumor cells, immune and inflammatory cells and tumor-related fibroblasts, etc.) and extracellular matrix components with high glutathione $(\mathrm{GSH})$, high hydrogen peroxide $\left(\mathrm{H}_{2} \mathrm{O}_{2}\right)$ and low oxygen $\left(\mathrm{O}_{2}\right)$ contents $[1$, 2]. The interaction between tumor cells and the complicated TME is responsible for drug resistance, limited therapeutic efficacy and invasion and metastasis after various treatments[3, 4]. It has been recognized that the modulation of the TME by promoting ROS (reactive oxygen species) and/or reducing ROS scavenging agents (e.g., GSH) could effectively ablate cancer cells by elevating intracellular oxidative stress[5-7]. Chemodynamic therapy (CDT) based on the Fenton reaction, which catalyses hydrogen dioxide $\left(\mathrm{H}_{2} \mathrm{O}_{2}\right)$ into highly toxic hydroxyl radicals $(\bullet \mathrm{OH})$ by iron ions, has emerged as a novel therapeutic approach for cancer through oxidative damage of lipids, proteins and DNA[8]. Recently, copper-based Fenton-like reactions with higher reaction rates in a broader $\mathrm{pH}$ range showed more possibilities for highly efficient CDT[9-13]. However, copper is a trace element in the body incapable of inducing sufficient therapeutic efficacy alone, which would induce toxicity to normal cells at high concentrations[14]. In order to enhance the anticancer efficacy and meanwhile compromise the side effects, it is a great urgent to integrate CDT with other therapeutic modalities.

Chemotherapy using toxic drugs has been one of the most pivotal methods to treat cancer over the past decades, while the clinical outcome is severely hindered by unsatisfactory antitumor efficacy and various side effects[15]. Among them, the anthracycline antibiotic doxorubicin (DOX) as an FDA-approved chemotherapeutic agent has been universally adopted to treat various cancers through intercalation between base pairs in the DNA helix to prevent DNA replication[16-18]. Moreover, it has been reported that DOX could activate adenine dinucleotide phosphate oxidases (NOXs) to catalyze adenine 
dinucleotide phosphate (NADPH) and $\mathrm{O}_{2}$ to produce NADP+ and superoxide[19]. Subsequently, in the presence of superoxide dismutase (SOD), superoxide would be disproportionate into oxygen and $\mathrm{H}_{2} \mathrm{O}_{2}[20$, 21], which could further fuel CDT. Hence, it would be of great promise for efficient cancer treatment by developing a biocompatible nanocarrier to specifically deliver and release DOX and copper ions into the tumor site for combined chemo and chemodynamic therapy.

Bovine serum albumin (BSA) is a biocompatible globulin in bovine serum, which has wide applications in nanomedicine[22-26]. The hydrophobic region on the protein is feasible for loading hydrophobic chemodrug such as DOX[27-30], while its abundant amino and cysteine groups tend to attach metal ions such as copper ions[31, 32]. However, the metal ions attached to albumin are prone to uncontrolled release without specificity, leading to unsatisfactory copper dosages for CDT and latent toxicity issues[33, 34]. Gallic acid (GA) is a polyphenol derived from tea that has been widely used in the pharmaceutical industry due to its economical and accessible extraction procedure[35]. Several nanoscale coordination polymers (NCPs) have been synthesized through the chelation reaction between metal ions and the carboxyl and phenolic hydroxyl groups of GA[36, 37].

Herein, we synthesize a novel nanomedicine (GA-Cu@BSA-DOX, GCBD NPs) based on BSA nanocarriers for combined chemo and chemodynamic cancer therapy. GA-Cu nanodots (GC NDs) are formed by the insitu coordination of GA and copper ions absorbed in BSA, which subsequently gather together by hydrophobic interactions upon the addition of doxorubicin (DOX), forming GCBD NPs with larger sizes. Under acidic conditions, the GCBD NPs would simultaneously release copper ions $\left(\mathrm{Cu}^{2+}\right)$ and DOX. The released $\mathrm{Cu}^{2+}$ could consume $\mathrm{GSH}$ and produce $\mathrm{Cu}^{+}$, which subsequently induces a Fenton-like reaction by catalysing a high content of $\mathrm{H}_{2} \mathrm{O}_{2}$ in the TME to generate highly cytotoxic hydroxyl radicals $(\cdot \mathrm{OH})$ for cancer cell death. Meanwhile, the enhanced drug delivery and specific DOX release based on the BSA nanocarrier would further kill tumor cells by inhibiting their DNA replication and produce more $\mathrm{H}_{2} \mathrm{O}_{2}$ to fuel CDT. The residual chemical groups in GCBD NPs could be stably labeled with radionuclide ${ }^{125}$ to evaluate the blood circulation and biodistribution behavior in mice[38-40]. Taking advantage of the efficient tumoral accumulation and specific release of DOX and $\mathrm{Cu}^{2+}, \mathrm{GCBD}$ NPs would strongly inhibit tumor growth by a synergistic effect. In this study, we designed a biocompatible nanoplatform to exploit TME characteristics for combined chemo and chemodynamic therapy, providing a novel strategy to eradicate tumors with high efficiency and specificity.

\section{Materials And Methods}

\subsection{Materials}

Bovine serum albumin (BSA) and 2',7'-dichlorofluorescin diacetate (DCFH-DA) were purchased from Sigma-Aldrich Co. Polyvinylpyrrolidone (PVP) was purchased from Alfa Aesar (China). Gallic acid (GA) and disodium terephthalate (TA) were purchased from Aladdin (Shanghai). Copper (II) chloride was purchased from Siuopharm Chemical Reagent Co. The GSH/GSSG assay kit, live/dead assay kit, calcein- 
AM and propidium iodide $(\mathrm{PI})$ were obtained from Beyotime Biotechnology. CCK-8 was obtained APExBIO. $\mathrm{HCl} \cdot \mathrm{DOX}$ was obtained from Meilunbio.

\subsection{Synthesis of nanomaterials}

PVP stabilized GA-Cu nanodots (GC NDs): $60 \mathrm{mg}$ PVP was dissolved in $8 \mathrm{~mL}$ deionized (DI) water under stirring, followed by dropwise addition of $1 \mathrm{~mL} \mathrm{CuCl} 2 \cdot 2 \mathrm{H}_{2} \mathrm{O}(10 \mathrm{mg} / \mathrm{mL})$ and $1 \mathrm{~mL} \mathrm{GA}(10 \mathrm{mg} / \mathrm{mL})$ for reaction overnight. The as-prepared GC NDs were then purified by centrifugation at $3500 \mathrm{rpm}$ for $10 \mathrm{~min}$ using ultrafiltration Millipore tubes (10 kDa) and washed three times with DI water.

GA-Cu@BSA nanodots (GCB NDs): 70 mg BSA was dissolved in $8 \mathrm{~mL}$ DI water under gentle stirring for 5 $\min$. Then, $1 \mathrm{~mL} \mathrm{CuCl} 2 \cdot 2 \mathrm{H}_{2} \mathrm{O}(10 \mathrm{mg} / \mathrm{mL})$ was added dropwise with constant stirring for $30 \mathrm{~min}$. After that, $1 \mathrm{~mL} 10 \mathrm{mg} / \mathrm{mL} \mathrm{GA}$ was added to the bottle with vigorous stirring for another $3 \mathrm{~h}$. After the reaction, the unreacted large particles were removed by centrifugation $(4000 \mathrm{rpm})$. Unreacted impurities were removed with an ultra-filtration (100 kDa, $3500 \mathrm{rpm})$ washed twice with DI water and then stored in a refrigerator at $4^{\circ} \mathrm{C}$ for further application.

GA-Cu@BSA-DOX nanoparticles (GCBD NPs): 10 mg DOX dissolved in $200 \mu \mathrm{L}$ DMSO was added dropwise into $1 \mathrm{~mL} 10 \mathrm{mg} / \mathrm{mL}$ GCB solution with vigorous stirring for $12 \mathrm{~h}$. After the reaction, the free DOX and DMSO were removed by ultra-filtration (100 kDa, $3500 \mathrm{rpm})$, washed with DI water for three times (until the UV absorption of DOX in the liquid below was less than 0.2 ) and then stored in a refrigerator at $4^{\circ} \mathrm{C}$ for further use.

\subsection{Characterization of nanomaterials}

The morphology and size of GA NDs, GCB NDs and GCBD NPs were characterized by TEM (Tecnai G2 spirit Bio Twin). Their physiological size and zeta-potential were tested by DLS (Zetasizer Nano ZS90). The absorbance spectra were tested by UV-Vis spectroscopy (GENESYS 10S Spectrophotometer). Fourier transform infrared (FT-IR) spectra were recorded by conventional Fourier infrared spectroscopy (NICOLET iS 50). XPS spectra were tested by Thermo Scientific K-Alpha. In order to test the physiological stability, GCB NDs and GCBD NPs were dissolved in PBS, water and DMEM, respectively $(n=3)$. Every other day, the solutions were photographed, and the size and PDI value were measured by DLS for 7 days.

\subsection{Drug loading and release behavior}

Drug loading capacity of GCB: In order to test the ability of GCB NDs to load drugs, $10 \mathrm{mg}$ GCB NDs was dissolved in $1 \mathrm{~mL}$ DI water. Then, DOX solutions with different concentrations $(20 \mathrm{mg} / \mathrm{mL}, 10 \mathrm{mg} / \mathrm{mL}, 5$ $\mathrm{mg} / \mathrm{mL}, 2.5 \mathrm{mg} / \mathrm{mL}$ and $1.25 \mathrm{mg} / \mathrm{mL}$ ) were added to GCB ND solutions for reaction overnight. After the reaction, the free DOX and DMSO were removed by ultrafiltration (100 kDa, $3500 \mathrm{rpm})$.

Release of DOX: In order to test the DOX release behavior, $10 \mathrm{mg}$ GCBD NPs was dissolved into $2 \mathrm{~mL}$ DI water in a dialysis bag, which was put into bottles containing $15 \mathrm{~mL}$ PBS ( $\mathrm{pH}=5.6$ or 7.4$)$ for stirring (3 parallel samples were set in each group). At $30 \mathrm{~min}, 1 \mathrm{~h}, 2 \mathrm{~h}, 4 \mathrm{~h}, 6 \mathrm{~h}, 12 \mathrm{~h}$ and $24 \mathrm{~h}$ after stirring, $1 \mathrm{~mL}$ of 
the solutions outside the dialysis bag was removed to record the absorbance intensity at $500 \mathrm{~nm}$ by UVVis and then put back to the system.

Release of copper ions: The release of $\mathrm{Cu}^{2+}$ from GCBD NPs was detected by Copper Determination Kit (Beyotime Biotechnology). Generally, $10 \mathrm{mg}$ GCBD NPs was dissolved in $2 \mathrm{~mL}$ DI water, which was then put into dialysis tube with PBS ( $\mathrm{pH} 5.6$ and $\mathrm{pH} 7.4$ ) (3 parallel samples were set in each group) outside, $10 \mu \mathrm{L}$ was taken at intervals of $5 \mathrm{~min}, 30 \mathrm{~min}, 1 \mathrm{~h}, 2 \mathrm{~h}, 6 \mathrm{~h}, 12 \mathrm{~h}, 24 \mathrm{~h}$. The same volume of PBS solution was then added to the reaction system.

\subsection{Labeling Cy5.5 and ${ }^{125}$ I onto GCBD NPs}

Cy5.5 labeling: $10 \mathrm{mg}$ GCB NDs and GCBD NPs were dissolved in $1 \mathrm{~mL}$ of DI water. Subsequently, $10 \mu \mathrm{L}$ aminated Cy 5.5 was added into the solution and stirred for $2 \mathrm{~h}$ under dark conditions. Excess Cy 5.5 was removed with $10 \mathrm{kDa}$ (for Cy5.5-GCB) and $100 \mathrm{kDa}$ (for Cy5.5-GCBD) ultra-filtration tube and the products were stored at $4^{\circ} \mathrm{C}$ in a dark environment for further use.

125 I labeling: $2.21 \mathrm{mg} \mathrm{1,3,4,6-Tetrachloro-3a,6a-diphenyl-glycouril} \mathrm{(Idongen)} \mathrm{was} \mathrm{dissolved} \mathrm{into} 600 \mu \mathrm{L}$ chloroform with chloroform and later blow-dried with nitrogen. Then, $2 \mathrm{mg} / \mathrm{mL}$ GCBD NPs were mixed with $500 \mu \mathrm{Ci} 125 \mathrm{I}$, and then the mixture was mixed with blow-dried Idongen for 20 min with shaking. After the reaction, the mixture was washed three times with $100 \mathrm{kDa}$ ultra-filtration tube.

Radiolabeling stability: The radiolabeling stability of ${ }^{125}$ I labeled GCBD NPs was then evaluated by

incubating ${ }^{125}$ I @GCBD NPs in serum or PBS for $48 \mathrm{~h}$. The unlabeled radionuclides were washed with 100 $\mathrm{kDa}$ ultra-filtration tube for several times. The residual nuclide dose in the solution at different time points was detected by gamma counter (PerkinElmer) for radiostability.

\subsection{Detection of hydroxyl radical and GSH}

Generation of $\cdot \mathrm{OH}$ catalysed by GCBD NPs: GCBD NPs $(100 \mu \mathrm{g} / \mathrm{ml}$ for GCB NDs) were incubated with disodium terephthalate (TA) (purchased from Aladdin) and $\mathrm{H}_{2} \mathrm{O}_{2}$ on a shaking table at $350 \mathrm{rpm}$ and $37^{\circ} \mathrm{C}$ for $3 \mathrm{~h}$. The final concentrations of TA and $\mathrm{H}_{2} \mathrm{O}_{2}$ in the reaction system were $1.5 \mathrm{mM}$ and $2 \mathrm{mM}$, respectively. TA+ $\mathrm{H}_{2} \mathrm{O}_{2}, \mathrm{TA}+\mathrm{GCBD} N \mathrm{NP}$, and $\mathrm{H}_{2} \mathrm{O}_{2}+\mathrm{GCBD}$ NPs were used as controls. The fluorescence emission peaks at $435 \mathrm{~nm}(\lambda \mathrm{ex}=315 \mathrm{~nm})$ were detected by fluorescence spectra of all samples.

Depletion of intracellular GSH: To detect the depletion of GSH by GCB NDs and GCBD NPs at the cellular level, $4 T 1$ cells were cultured in 6 -well plates for $24 \mathrm{~h}$. Then, cells were cocultured with GCB NDs $(n=3)$ and GCBD NPs $(n=3)$. The blank group without nanomaterials was used as a control, respectively. After $24 \mathrm{~h}$, cells were collected by centrifugation at $1000 \mathrm{rpm}$. The GSH content in each group was detected according to the instructions of the GSH/GSSG assay kit (Beyotime Biotechnology).

\subsection{Cellular experiments}

Cytotoxicity: CCK-8 assay was used to detect the biosafety and cytotoxicity of NPs. Generally, 4T1 cells and human umbilical vein epithelial cells (HUVECs), which were cultured in high glucose DMEM medium 
with $10 \%$ fetal bovine serum and $1 \%$ penicillin-streptomycin in a humidified atmosphere containing $5 \%$ $\mathrm{CO}_{2}$ at $37^{\circ} \mathrm{C}$, were placed in 96-well plates and cultured in a cell incubator for $24 \mathrm{~h}$. For biosafety, GCB NDs with different concentration gradients were added to the HUVEC plates. For cytotoxicity, GCB NDs, DOX, GCBD NPs with different concentration gradients and blanks were cocultured with 4T1 cells. After $24 \mathrm{~h}$ of culture, the relative cell viability was tested by CCK-8 assay.

Detection of cellular $\cdot 0 \mathrm{H}: 4 \mathrm{~T} 1$ cells were treated with DCFH-DA $(10 \mu \mathrm{M}$, dispersed in DMEM medium) for 20 mins. After washing with PBS for three times, samples were then separated into three groups: blank group cultured only with DMEM medium, experimental group incubated with GCB NDs $(20 \mu \mathrm{g} / \mathrm{mL})$ and GCBD NPs $(6 \mu \mathrm{g} / \mathrm{mL}$ for DOX) for $3 \mathrm{~h}$. Then, all samples were imaged by fluorescence microscope (OLYMPUS IX73).

Dead and live cell staining: In order to display the cytotoxicity of the materials more intuitively, live and dead staining was used to further verify apoptosis. 4T1 cells were cocultured with DOX $(10 \mu \mathrm{g} / \mathrm{ml}), \mathrm{GCB}$ NDs $(200 \mu \mathrm{g} / \mathrm{ml})$, and GCBD NPs $(10 \mu \mathrm{g} / \mathrm{ml}$ in DOX concentration) for $24 \mathrm{~h}$, respectively. Afterwards, cells in different groups were stained with Calcein-AM $(2 \mu \mathrm{M})$ and $\mathrm{PI}(4.5 \mu \mathrm{M})$ for 15 mins and then observed by fluorescence microscope (OLYMPUS IX73).

Cellular uptake of DOX: 4T1 cells were incubated with GCBD NPs at $1 \mu \mathrm{g} / \mathrm{mL}$ doxorubicin and free DOX (1 $\mu \mathrm{g} / \mathrm{mL}$ ) in 24-well plates with high glucose medium. After incubation at different time points for 1, 6, 12, and $24 \mathrm{~h}$, the medium was removed. Then, the $4 \mathrm{~T} 1$ cells were washed with PBS for 3 times and fixed with $4 \%$ paraformaldehyde for $15 \mathrm{~min}$. Then, the nuclei were stained with 4',6-diamidino-2-phenylindole (DAPI) for $15 \mathrm{~min}$. After that, the cells were washed with PBS for 3 times and finally photographed with confocal laser microscope (FV1200).

Cell apoptosis: 4T1 cells were first incubated in 6-well plates for $24 \mathrm{~h}$. Then, cells were incubated with GCB NDs or GCBD NPs for $24 \mathrm{~h}$, and the group without materials was used as the control. Cells were collected by centrifugation at $1000 \mathrm{rpm}$ and then stained with Annexin V-FITC and PI at $20-25^{\circ} \mathrm{C}$ for 20 min. Finally, the apoptosis of $4 \mathrm{~T} 1$ cells was detected by flow cytometry (BD FACSVERSE).

\subsection{Blood circulation and biodistribution}

Blood circulation of GCBD NPs: GCBD NPs labeled with ${ }^{125}$ (125I@GCBD NPs) were injected into tumorbearing BALB/c mice $(n=3)$ through the tail vein for blood circulation and tissue distribution, respectively. For blood circulation, $10 \mu \mathrm{L}$ of blood was collected from the orbit of mice, and the blood weight was recorded. The radiation intensity of blood was measured by gamma counter (PerkinElmer) at $5 \mathrm{~min}, 15$ $\min , 30 \mathrm{~min}, 1 \mathrm{~h}, 2 \mathrm{~h}, 4 \mathrm{~h}, 10 \mathrm{~h}$ and $24 \mathrm{~h}$.

Biodistribution of GCBD NPs: GCBD NPs were injected into BALB-c mice $(n=3)$ through the tail vein to study the distribution of the material in vivo by measuring the intensity of radioactivity of $125 \mathrm{I}$. The radiation intensity in the heart, liver, spleen, lung, kidney and tumors of the sacrificed mice was measured by gamma counter (PerkinElmer) after the injection of 125I@GCBD NPs for $4 \mathrm{~h}$ and $24 \mathrm{~h}$, respectively. 
In vivo imaging: In order to reflect the enrichment of materials in mice more intuitively, GCB NDs and GCBD NPs were labeled with Cy5.5 and then injected into mice that were randomly divided into 2 groups $(n=5)$ through the tail vein. Then, the mice in each group were photographed by IVIS (PerkinEimer) at $0 \mathrm{~h}$, $1 \mathrm{~h}, 4 \mathrm{~h}, 12 \mathrm{~h}$ and $24 \mathrm{~h}$. After injection for $24 \mathrm{~h}$, all mice were sacrificed to collect their hearts, livers, spleens, lungs, kidneys and tumors.

\subsection{Combined chemo/chemodynamic therapy of tumor}

In order to verify the effect of tumor therapy in vivo, 4T1 cells were injected into the back of BALB-c mice to establish $4 \mathrm{~T} 1$ tumor-bearing mouse models. When the tumor volume reached approximately $50-100$ $\mathrm{mm}^{3}$, the mice were randomly divided into 4 groups ( $\mathrm{n}=6$ ): (1) PBS, (2) DOX (5 mg kg-1), (3) GCB NDs (18.72 mg kg-1), and (4) GCBD NPs (5 mg kg-1 of DOX) and respectively injected with specific solutions through the tail vein. On day 7 post treatment, one mouse in each group was randomly sacrificed, and the tumor was subjected to TUNEL staining to test the therapeutic effect of the drugs. One mouse in each group was randomly sacrificed for H\&E-stained slices of its heart, liver, spleen, lung, kidney and tumor after the treatment to test whether drugs have obvious toxic and side effects on normal tissues and 4T1 tumors of mice. The tumor volume and the weight of the mice were recorded every other day for 14 days. All the mice were sacrificed after the treatment, and the 4T1 tumors were removed, weighed and photographed.

\section{Results And Discussion}

\subsection{Synthesis and characterization of BSA nanocarriers}

The synthesis procedure of GA-Cu@BSA-DOX nanoparticles (GCBD NPs) for TME-responsive chemo/chemodynamic cancer therapy is illustrated in Scheme 1. Briefly, copper ions were attached onto bovine serum albumin (BSA) through electrostatic interactions, which were then solidified by gallic acid (GA) chelation in situ to form GA-Cu@BSA nanodots (GCB NDs). Transmission electron microscope (TEM) imaging showed that GCB NDs were evenly distributed with an average size of $8.1 \mathrm{~nm}$, which was larger than that of PVP-stabilized GA-Cu nanodots (GC NDs), indicating that BSA functioned as the template rather than surfactant during the reaction (Fig. 1a\&S1). The chemical groups in GCB NDs were analyzed by Fourier transform infrared (FT-IR) spectrometry (Fig. S2). The characteristic peaks of GCB NDs were similar to those of BSA, indicating the presence of BSA in the GCB NDs. Meanwhile, the characteristic peaks of $3290 \mathrm{~cm}^{-1}$ (O-H stretching) and $1030 \mathrm{~cm}^{-1}$ (O-H of the carboxylic group) for GA vanished in the FT-IR spectra of GCB NDs, revealing that the copper ions were chelated with the phenolic hydroxyl and carboxyl groups of GA.

\subsection{DOX loading onto GCB NDs}

GCB NDs were then evaluated as BSA nanocarriers to load FDA-approved chemodrug (DOX) at different mass ratios, achieving GCBD NPs through hydrophobic interactions by stirring overnight in the dark. The 
DOX loading efficiency gradually increased as the mass ratio of DOX to GCB NDs increased until a plateau of approximately $30.12 \%$ for GCBD NPs (Fig. S3). TEM images of GCBD NPs showed that DOX encapsulation resulted in an enlarged average size of $40.1 \mathrm{~nm}$ (Fig. 1b). Dynamic light scattering (DLS) determined that the average hydrodynamic size increased from $10 \mathrm{~nm}$ for GCB NDs to $60 \mathrm{~nm}$ for GCBD NPs (Fig. 1c), which was consistent with the TEM results, while the slightly larger hydrodynamic size was reasonable due to the hydration of nanomaterials in solution. Since DOX was positively charged, the negatively charged GCB NDs turned into positively charged GCBD NPs after DOX loading (Fig. 1d). In addition, the absorbance spectra of GCBD NPs demonstrated the characteristic absorbance peaks of BSA at $275 \mathrm{~nm}$, and DOX redshifted to about $500 \mathrm{~nm}$, preliminarily confirming that DOX was loaded onto the BSA nanocarriers (Fig. 1e). Furthermore, in contrast to GCB NDs, the FT-IR spectra of GCBD NPs showed the characteristic peaks of DOX at $3421 \mathrm{~cm}^{-1}$ (O-H stretching vibration peak), $1652 \mathrm{~cm}^{-1}$ (C-C ring tension stretching vibration peak), $1618 \mathrm{~cm}^{-1}$ ( $\mathrm{C}=\mathrm{O}$ stretching vibration peak), $1406 \mathrm{~cm}^{-1}$ (C-H bending vibration peak), $1284 \mathrm{C}$ (C=N stretching vibration peak), $1211 \mathrm{~cm}^{-1}$ (C-O-C stretching vibration peak) and $987 \mathrm{~cm}^{-1}$ $(\mathrm{N}-\mathrm{H}$ stretching vibration peak), validating the successful DOX loading onto the BSA nanocarriers (Fig. 1f).

To evaluate the elemental composition and valence state of the as-prepared nanomaterials, $\mathrm{X}$-ray photoelectron spectroscopy (XPS) was conducted after complete purification (Fig. S4). All GC NDs, GCB NDs and GCBD NPs showed the characteristic peaks of C 1s (284.8 eV), N 1s (399.8 eV), 0 1s (531.7 eV), and $\mathrm{Cu} 2 \mathrm{p}(934.4 \mathrm{eV})$, indicating the successful chelation and solidication of copper ions by GA. The higher ratio of $\mathrm{N}$ in GCB NDs than in GC NDs was due to the higher $\mathrm{N}$ content in BSA than in PVP, while the higher ratio of $C$ and $O$ in GCBD NPs than in GCB NDs stemmed from the encapsulation of DOX. To further determine the valence state of copper, the corresponding high-resolution $\mathrm{Cu} 2 \mathrm{p}$ spectra were obtained and analyzed. The two dominant peaks at 934.3 and $954.2 \mathrm{eV}$ could be ascribed to $\mathrm{Cu} 2 \mathrm{p} 3 / 2$ and $\mathrm{Cu} 2 \mathrm{p} 1 / 2$ of $\mathrm{Cu}^{2+}$, while the satellite peaks in the range of $938-948 \mathrm{eV}$ were testified to be the typical peaks of $\mathrm{Cu}^{2+}$ (Fig. S5). The copper content in the GCBD NPs was $0.94 \pm 0.24 \mathrm{wt} \%$ as determined by inductively coupled plasma-optical emission spectrometry (ICP-OES), which was in accordance with that semiquantified from the XPS results.

\section{3 $\mathrm{pH}$-sensitive release of therapeutic agents and production of hydroxyl radicals}

The promise of GCBD NPs for TME-triggered therapy was then evaluated. Considering the relatively acidic environment in tumors $(\mathrm{pH}=5.6)$, we investigated the release profile of copper ions and DOX from GCBD NPs at different $\mathrm{pH}$ values. The time-dependent release of DOX from GCBD NPs was evaluated by testing the absorbance spectra of solutions outside the dialysis bags (Fig. 1g). At pH 7.4, less than $7.2 \%$ of DOX was released from GCBD NPs after incubation for $24 \mathrm{~h}$. Meanwhile, at pH 5.6, the DOX release profile of GCBD NPs showed a sharp increase in the first $2 \mathrm{~h}$ and achieved as high as $47.3 \%$ after $24 \mathrm{~h}$, indicating the promise of GCBD NPs for TME-responsive chemotherapy. 
The time-dependent release of copper ions from GCBD NPs was evaluated by ICP-OES (Fig. 1h). GCBD NPs could release about $92.7 \%$ of copper ions under acidic conditions and less than $9 \%$ under neutral conditions after $24 \mathrm{~h}$. The significantly greater release of copper ions under acidic conditions is promising for the TME-sensitive release of sufficient copper ions, which could catalyze Fenton-like reactions to produce cytotoxic $\cdot \mathrm{OH}$ for chemodynamic therapy (CDT). Subsequently, the concentration of $\cdot \mathrm{OH}$ produced from $\mathrm{H}_{2} \mathrm{O}_{2}$ at a tumoral concentration ( $1 \mathrm{mM}$ ) was investigated by disodium terephthalate (TA), which could react with $\mathrm{OH}$ to produce $\mathrm{TAOH}$ with a fluorescence peak at $435 \mathrm{~nm}$ ( $\lambda$ ex=315 nm) (Fig. 1i). Although a slight signal of TAOH appeared in free $\mathrm{H}_{2} \mathrm{O}_{2}$, a much stronger fluorescence intensity emerged after incubating GCBD NPs with $\mathrm{H}_{2} \mathrm{O}_{2}$ for $3 \mathrm{~h}$, suggesting the capability of GCBD NPs for specific CDT of cancer cells.

Furthermore, the physiological stability of the as-prepared nanomaterials was evaluated. GC NDs, GCB NDs and GCBD NPs were lyophilized and redispersed in water, resulting in clear solutions with specific colours (Fig. S6), which is promising for clinical translation. The GCB NDs and GCBD NPs in different solutions (water, PBS and DMEM medium) were monitored for 7 days, showing steady hydrodynamic diameter and PDI with negligible agglomeration or precipitation (Fig. S7). These results all together supported the further evaluation of BSA-based nanomaterials for biomedical applications.

\subsection{Chemo/chemodynamic therapy of cancer cells}

We then evaluated the promise of GCBD NPs for combined chemo/chemodynamic therapy in vitro (Fig. 2a). Before conducting chemo/chemodynamic therapy of cancer cells, we first assessed the biocompatibility of the BSA nanocarriers to normal cells. After incubation with GCB nanodots for $24 \mathrm{~h}$, the relative cell viability of human umbilical vein epithelial cells (HUVECs) was over $75 \%$, even at the highest concentration of $200 \mu \mathrm{g} / \mathrm{mL}$ (Fig. 2b), encouraging us to further study the therapeutic efficacy of these nanomaterials for cancer cells. For mouse breast cancer cells (4T1 cells), GCB nanodots showed little toxicity at concentrations ranging from 0 to $100 \mu \mathrm{g} / \mathrm{mL}$, while $38 \%$ of cells died at a concentration of 200 $\mu \mathrm{g} / \mathrm{mL}$ (Fig. 2c). Meanwhile, GCBD NPs showed a more significant lethal effect on cancer cells than GCB nanodots, resulting in less than $62 \%$ relative viability of $4 T 1$ cells at the highest concentration ([DOX] $=20$ $\mu \mathrm{g} / \mathrm{mL}$, [GCB] $=200 \mu \mathrm{g} / \mathrm{mL}$ ) (Fig. 2c). Confocal imaging of CA/PI-stained $4 \mathrm{~T} 1$ cells also revealed maximum cancer cell death after incubation with GCBD NPs, indicating the potent efficacy of combined chemo- and chemodynamic therapy of cancer cells (Fig. 2d\&S8a). Flow cytometry was then conducted to analyze the cell death pathway, showing that the apoptosis rate of cells after GCBD NP treatment was significantly higher than those treated with PBS or GCB NDs and even higher than those treated with free DOX (Fig. 2e\&S8b), which was consistent with the cytotoxicity and confocal results.

To trace the mechanism for the stronger cytotoxicity of GCBD NPs to cancer cells, we first evaluated the cellular uptake of DOX by confocal imaging and flow cytometry. Confocal imaging showed that the fluorescence of free DOX was brighter with incubation time, especially in the cell nuclei (Fig. 3a). Meanwhile, for GCBD NP-treated cells, DOX fluorescence quickly appeared in the lysosome after $4 \mathrm{~h}$ and ultimately in the nuclei at $24 \mathrm{~h}$ of incubation (Fig. 3b), suggesting the release of DOX in acidic cellular 
lysosomes for entering nuclei to bind with DNA. Flow cytometry (Fig. 3c-d), consistent with the semiquantitative analysis (Fig. 3e-f), showed stronger DOX fluorescence intensity in GCBD NP-treated cells than in cells treated with free DOX at each time point. These results indicated more cellular uptake of DOX with the delivery of BSA nanocarriers, which is beneficial for enhancing chemotherapy efficacy.

Considering the capability of GCBD NPs to catalyze $\mathrm{H}_{2} \mathrm{O}_{2}$ to $\cdot \mathrm{OH}$, DCFH-DA was then applied as the fluorescent probe to detect $\cdot \mathrm{OH}$ for chemodynamic therapy, showing much stronger green fluorescence in GCB NDs and GCBD NPs than in the control group (Fig. 4a-b). Since the presence of GSH would compromise the toxicity of $\cdot \mathrm{OH}$ to cells, we then examined the cellular concentration of GSH by a GSH/GSSG detection kit. Compared with the blank group, GCB NDs and GCBD NPs with copper ions could effectively consume GSH (Fig. 4c), resulting in chemically inert GSSG (Fig. 4d). These results verified that GCBD NPs could not only catalyze $\mathrm{H}_{2} \mathrm{O}_{2}$ into cytotoxic reactive oxygen species but also modulate the high expression of GSH in GSSH, suggesting promise for TME-responsive chemodynamic therapy.

\subsection{Blood circulation and biodistribution of GCBD NPs in mice}

To evaluate the blood circulation and biodistribution of GCBD NPs in mice, radionuclide ${ }^{125}$ I was labeled onto GCBD NPs, showing ideal labeling stability in both serum and PBS within $48 \mathrm{~h}$ (Fig. S9). After intravenous administration into healthy Balb-c mice, GCBD NPs demonstrated a circulation half-life of $1.05 \pm 0.18 \mathrm{~h}$ (Fig. $5 \mathrm{a}$ ). Meanwhile, the ex vivo radionuclide intensity showed that the tumoral accumulation of GCBD NPs reached $8.7 \%$ at $4 \mathrm{~h}$ and $3.5 \%$ at $24 \mathrm{~h}$ post i.v. injection (Fig. $5 \mathrm{~b}$ ).

On the other hand, the fluorescence imaging of Cy5.5-labeled GCB NDs and GCBD NPs in tumor-bearing mice exhibited the highest tumoral accumulation at $4 \mathrm{~h}$ post administration (Fig. 5c). Besides, GCBD NPs showed longer retention time in tumors due to their larger size. Moreover, the fluorescence imaging of mice, consistent with the radionuclide intensity of major organs, demonstrated the gradually decreased signal of GCBD NPs in the liver, kidney and spleen over time, preliminarily indicating the rapid metabolism of GCBD NPs in mice. These results together promised GCBD NPs for combined therapy of tumors.

\subsection{Chemo/chemodynamic therapy of tumor}

The 4T1 tumor-bearing mice were randomly divided into four groups (6 mice in each group) when the size of inoculated tumors reached $50 \mathrm{~mm}^{3}$, which were i.v. injected with PBS, free DOX, GCB NDs or GCBD NPs. The tumoral size of the mice was monitored by caliper over the next 14 days. Although free DOX or GCB could moderately restrain the tumor volume, GCBD NPs could inhibit the growth of tumors more effectively due to the combination of chemodrug and CDT agents (Fig. 6a). The tumors excised from mice after 14 days also demonstrated the best therapeutic efficacy by combining chemo/chemodynamic therapy based on GCBD NPs (Fig. 6b\&S10). Hematoxylin and eosin (H\&E) and TUNEL stained tumor 
slices revealed the most obvious cellular necrosis based on GCBD NPs compared with other groups (Fig. 6c), further confirming the synergistic effect of combined chemo/chemodynamic therapy.

The body weight of mice slightly declined during the first two days after systematic administration of GCBD NPs but recovered to a level as high as that of the PBS group after 14 days, preliminarily suggesting the biocompatibility of GCBD NPs to mice (Fig. S11). Furthermore, the mice were sacrificed at 14 days post-treatment, and major organs and tissues were collected and stained with H\&E (Fig. S12). The histopathological analysis showed negligible inflammation or injury in each organ, further indicating the biosafety of GCBD NPs in mice during the monitoring period.

\section{Conclusions}

In summary, we synthesized BSA-based nanocarriers named GCBD NPs for combining chemotherapy and CDT therapy of cancer. The prepared GCBD NPs with regular morphology and size showed high physiological stability for at least one week. Under acidic conditions, GCBD NPs specifically released 90\% of copper ions, which could convert GSH into GSSG at the cellular level and catalyze $\mathrm{H}_{2} \mathrm{O}_{2}$ into cytotoxic hydroxyl radicals for CDT. Meanwhile, the cellular uptake of DOX was enhanced by the delivery of GCBD NPs, which further entered cellular nuclei by acid-enhanced release to induce more potent cancer cell death. The chemo and chemodynamic therapy together induced the most apoptosis of 4T1 cells, as revealed by CCK-8, immunofluorescence imaging and flow cytometry. With the help of radionuclide ${ }^{125}$, labeling, the pharmacokinetics of GCBD NPs were monitored, showing a blood circulation half-life of 1.05 $\mathrm{h}$ and efficient tumoral accumulation, especially at $4 \mathrm{~h}$ post i.v. injection. After systematic administration, GCBD NPs showed the most effective inhibition of 4T1 tumor growth as compared to other groups, whose necrosis was further validated by TUNEL and H\&E-stained tumor slices. Furthermore, H\&E-stained slices of the major organs obtained 14 days after treatments preliminarily suggested that GCBD NPs had no obvious toxicity or side effects on mice. Our results indicated that combined chemotherapy and CDT therapy based on biocompatible nanocarriers is a promising cancer treatment strategy to achieve potent therapeutic efficacy with negligible adverse effects, which could be further extended to various nanoplatforms incorporating other metal ions and drugs.

\section{Declarations}

\section{Funding}

This work was supported by the National Natural Science Foundation of China (31822022, U1932208, 32171382, 31671797, 32000990), Natural Science Foundation of Jiangsu Province (BK20190946), Scientific Research Project of Anhui Provincial Higher Education Institutes (KJ2020A0375), Anhui Provincial Higher Education Institutes (KJ2020a0375), and Anhui Polytechnic University (xjky2020064).

\section{Availability of data and materials}

All data used to generate these results is available in the main text and supporting information. 


\section{Ethics approval and consent to participate}

All animal studies were approved by the Animal Ethics and Welfare Committee of Soochow University.

\section{Consent for publication}

All authors agree to be published.

\section{Competing interests}

The authors declare no conflict of interests.

\section{References}

1. Henke E, Nandigama R, Ergün S. Extracellular Matrix in the Tumor Microenvironment and Its Impact on Cancer Therapy. Front Mol Biosci. 2020;6:160.

2. Hinshaw DC, Shevde LA. The Tumor Microenvironment Innately Modulates Cancer Progression. Cancer Res. 2019;79(18):4557-66.

3. Ji RC. Hypoxia and lymphangiogenesis in tumor microenvironment and metastasis. Cancer Lett. 2014;346(1):6-16.

4. Stapleton S, Jaffray D, Milosevic M. Radiation effects on the tumor microenvironment: Implications for nanomedicine delivery. Adv Drug Deliv Rev. 2017;109:119-30.

5. Wang X, Zhong X, Liu Z, Cheng L. Recent progress of chemodynamic therapy-induced combination cancer therapy. Nano Today. 2020;35:100946.

6. Xin J, Deng C, Aras O, Zhou M, Wu C, An F. Chemodynamic nanomaterials for cancer theranostics. J Nanobiotech. 2021;19(1):192.

7. Pei P, Sun C, Tao W, Li J, Yang X, Wang J. ROS-sensitive thioketal-linked polyphosphoesterdoxorubicin conjugate for precise phototriggered locoregional chemotherapy. Biomaterials. 2019;188:74-82.

8. Zhang C, Bu W, Ni D, Zhang S, Li Q, Yao Z, Zhang J, Yao H, Wang Z, Shi J. Synthesis of Iron Nanometallic Glasses and Their Application in Cancer Therapy by a Localized Fenton Reaction. Angew Chem Int Ed. 2016;55(6):2101-6.

9. Lu J, Jiang Z, Ren J, Zhang W, Li P, Chen Z, Zhang W, Wang H, Tang B. One-Pot Synthesis of Multifunctional Carbon-Based Nanoparticle-Supported Dispersed $\mathrm{Cu}(2+)$ Disrupts Redox Homeostasis to Enhance CDT, Angew Chem Int Ed (2021).

10. Sun S, Chen Q, Tang Z, Liu C, Li Z, Wu A, Lin H. Tumor Microenvironment Stimuli-Responsive Fluorescence Imaging and Synergistic Cancer Therapy by Carbon-Dot-Cu(2+) Nanoassemblies. Angew Chem Int Ed. 2020;59(47):21041-8.

11. Lin LS, Huang T, Song J, Ou XY, Wang Z, Deng H, Tian R, Liu Y, Wang JF, Liu Y, Yu G, Zhou Z, Wang S, Niu G, Yang HH, Chen X. Synthesis of Copper Peroxide Nanodots for H2O2 Self-Supplying 
Chemodynamic Therapy. J Am Chem Soc. 2019;141(25):9937-45.

12. Mestivier M, Li JR, Camy A, Frangville C, Mingotaud C, Benoît-Marquié F. J.A.-O. Marty, Copper-Based Hybrid Polyion Complexes for Fenton-Like Reactions, Chem-Eur J (2020) 1521-3765.

13. Tian H, Zhao S, Nice EC, Huang C, He W, Zou B, Lin J. A cascaded copper-based nanocatalyst by modulating glutathione and cyclooxygenase-2 for hepatocellular carcinoma therapy. J Colloid Interf Sci. 2022;607:1516-26.

14. Denoyer D, Clatworthy S, Cater M. Copper Complexes in Cancer Therapy. Metallo-Drugs. 2018;16:469-506.

15. Huang C-Y, Ju D-T, Chang C-F, Muralidhar Reddy P, Velmurugan BK. A review on the effects of current chemotherapy drugs and natural agents in treating non-small cell lung cancer. Biomed (Taipei). 2017;7(4):23-3.

16. Fu LH, Hu YR, Qi C, He T, Jiang S, Jiang C, He J, Qu J, Lin J, Huang P. Biodegradable ManganeseDoped Calcium Phosphate Nanotheranostics for Traceable Cascade Reaction-Enhanced Anti-Tumor Therapy. ACS Nano. 2019;13(12):13985-94.

17. An J, Hu YG, Cheng K, Li C, Hou XL, Wang GL, Zhang XS, Liu B, Zhao YD, Zhang MZ. ROS-augmented and tumor-microenvironment responsive biodegradable nanoplatform for enhancing chemosonodynamic therapy. Biomaterials. 2020;234:119761.

18. Jiang W, Luo X, Wei L, Yuan S, Cai J, Jiang X, Hu Y. The Sustainability of Energy Conversion Inhibition for Tumor Ferroptosis Therapy and Chemotherapy. Small. 2021;17(38):e2102695.

19. Kim T, Davis J, Zhang AJ, He X, Mathews ST. Curcumin activates AMPK and suppresses gluconeogenic gene expression in hepatoma cells. Biochem Biophys Res Commun. 2009;388(2):377-82.

20. Ling X, Wang H, Huang S, Xia F, Dresselhaus MS. The renaissance of black phosphorus. Proc Natl Acad Sci U S A. 2015;112(15):4523-30.

21. Dai Y, Yang Z, Cheng S, Wang Z, Zhang R, Zhu G, Wang Z, Yung BC, Tian R, Jacobson O, Xu C, Ni Q, Song J, Sun X, Niu G, Chen X. Toxic Reactive Oxygen Species Enhanced Synergistic Combination Therapy by Self-Assembled Metal-Phenolic Network Nanoparticles. Adv Mater. 2018;30(8):1704877.

22. Park T, Lee S, Amatya R, Cheong H, Moon C, Kwak HD, Min KA, Shin MC. ICG-Loaded PEGylated BSASilver Nanoparticles for Effective Photothermal Cancer Therapy. Int J Nanomedicine. 2020;15:545971.

23. Chen J, Chen Q, Liang C, Yang Z, Zhang L, Yi X, Dong Z, Chao Y, Chen Y, Liu Z. Albumin-templated biomineralizing growth of composite nanoparticles as smart nano-theranostics for enhanced radiotherapy of tumors. Nanoscale. 2017;9(39):14826-35.

24. Ding H, Yang D, Zhao C, Song Z, Liu P, Wang Y, Chen Z, Shen J. Protein-gold hybrid nanocubes for cell imaging and drug delivery. ACS Appl Mater Inter. 2015;7(8):4713-9.

25. Wen Y, Dong H, Wang K, Li Y, Li Y. Self-Templated, Green-Synthetic, Size-Controlled Protein Nanoassembly as a Robust Nanoplatform for Biomedical Application. ACS Appl Mater Inter. 2018;10(14):11457-66. 
26. Zhou B, Song J, Wang M, Wang X, Wang J, Howard EW, Zhou F, Qu J, Chen WR. BSA-bioinspired gold nanorods loaded with immunoadjuvant for the treatment of melanoma by combined photothermal therapy and immunotherapy. Nanoscale. 2018;10(46):21640-7.

27. Liu Y, Qiao Z, Gao J, Wu F, Sun B, Lian M, Qian J, Su Y, Zhu X, Zhu B. Hydroxyapatite-Bovine Serum Albumin-Paclitaxel Nanoparticles for Locoregional Treatment of Osteosarcoma. Adv Healthc Mater. 2021;10(2):e2000573.

28. Leinwand JC, Bates GE, Allendorf JD, Chabot JA, Lewin SN, Taub RN. Body surface area predicts plasma oxaliplatin and pharmacokinetic advantage in hyperthermic intraoperative intraperitoneal chemotherapy. Ann Surg Oncol. 2013;20(4):1101-4.

29. Huang H, Yang DP, Liu M, Wang X, Zhang Z, Zhou G, Liu W, Cao Y, Zhang WJ, Wang X. pH-sensitive Au-BSA-DOX-FA nanocomposites for combined CT imaging and targeted drug delivery. Int $\mathrm{J}$ Nanomedicine. 2017;12:2829-43.

30. Liang J, Jin X, Chen B, Hu J, Huang Q, Wan J, Hu Z, Wang B. Doxorubicin-loaded pH-responsive nanoparticles coated with chlorin e6 for drug delivery and synergetic chemo-photodynamic therapy. Nanotechnology. 2020;31(19):195103.

31. Ma X, Ren X, Guo X, Fu C, Wu Q, Tan L, Li H, Zhang W, Chen X, Zhong H, Meng X. Multifunctional ironbased Metal-Organic framework as biodegradable nanozyme for microwave enhancing dynamic therapy. Biomaterials. 2019;214:119223.

32. Li X-F, Yang Y-Q, Li Y-X, Yang H-X, Zhao W-F, Meng X-R. Synthesis, crystal structure, and BSA binding studies of new $\mathrm{Co}(\mathrm{II})$ and $\mathrm{Ni}(\mathrm{II})$ complexes of 2-(hydroxymethyl)-1 H-imidazole-4,5-dicarboxylate. Inorg Chim Acta. 2020;505:119469.

33. Liu J, Sun L, Li L, Zhang R, Xu ZP. Synergistic Cancer Photochemotherapy via Layered Double Hydroxide-Based Trimodal Nanomedicine at Very Low Therapeutic Doses. ACS Appl Mater Inter. 2021;13(6):7115-26.

34. Chang Y, Jiang J, Chen W, Yang W, Chen L, Chen P, Shen J, Qian S, Zhou T, Wu L, Hong L, Huang Y, Li F. Biomimetic metal-organic nanoparticles prepared with a 3D-printed microfluidic device as a novel formulation for disulfiram-based therapy against breast cancer. Appl Mater Today. 2020;18:100492.

35. Ashrafizadeh M, Zarrabi A, Mirzaei S, Hashemi F, Samarghandian S, Zabolian A, Hushmandi K, Ang HL, Sethi G, Kumar AP, Ahn KS, Nabavi N, Khan H, Makvandi P, Varma RS. Gallic acid for cancer therapy: Molecular mechanisms and boosting efficacy by nanoscopical delivery. Food Chem Toxicol. 2021;157:112576.

36. El-Ghareb WI, Swidan MM, Ibrahim IT, Abd El-Bary A, Tadros MI, Sakr TM. (99m)Tc-doxorubicinloaded gallic acid-gold nanoparticles ((99m)Tc-DOX-loaded GA-Au NPs) as a multifunctional theranostic agent. Int J Pharm. 2020;586:119514.

37. Zheng H, Ma B, Shi Y, Dai Q, Li D, Ren E, Zhu J, Liu J, Chen H, Yin Z, Chu C, Wang X, Liu G. Tumor microenvironment-triggered MoS2@GA-Fe nanoreactor: A self-rolling enhanced chemodynamic therapy and hydrogen sulfide treatment for hepatocellular carcinoma. Chem Eng J. 2021;406:126888. 
38. Yi X, Zhou H, Zhang Z, Xiong S, Yang K. X-rays-optimized delivery of radiolabeled albumin for cancer theranostics. Biomaterials. 2020;233:119764.

39. Tian L, Chen Q, Yi X, Wang G, Chen J, Ning P, Yang K, Liu Z. Radionuclide I-131 Labeled AlbuminPaclitaxel Nanoparticles for Synergistic Combined Chemo-radioisotope Therapy of Cancer. Theranostics. 2017;7(3):614-23.

40. Pei P, Liu T, Shen W, Liu Z, Yang K. Biomaterial-mediated internal radioisotope therapy. Mater Horiz. 2021;8(5):1348-66.

\section{Scheme}

Scheme 1 is available in the Supplemental Files section

\section{Figures}

a

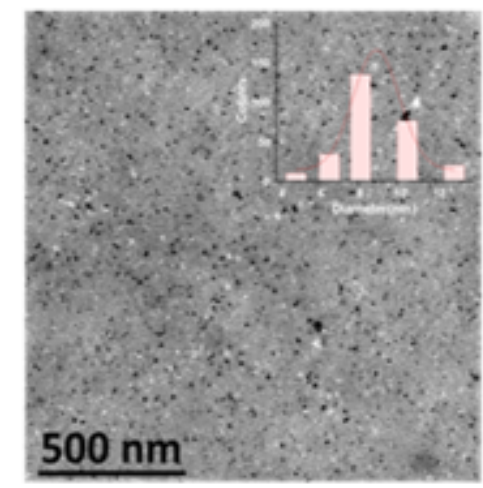

d

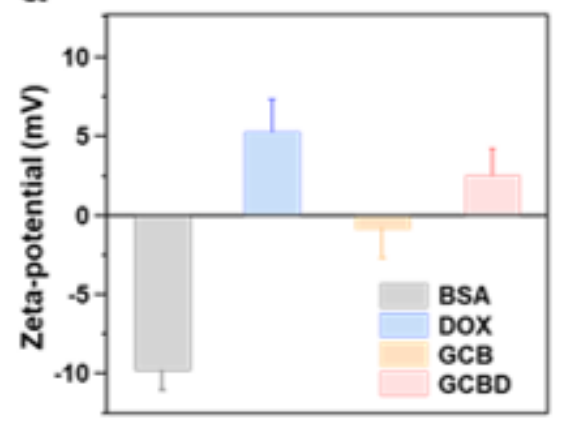

g

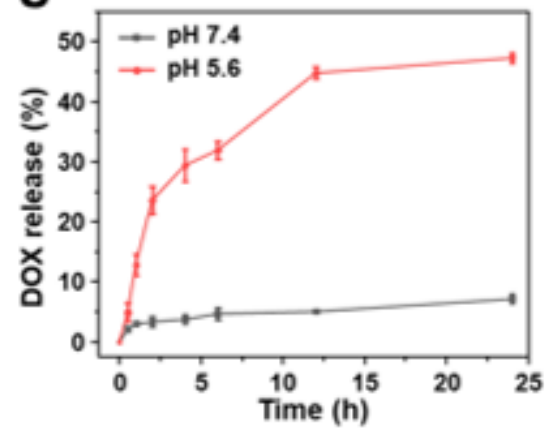

b

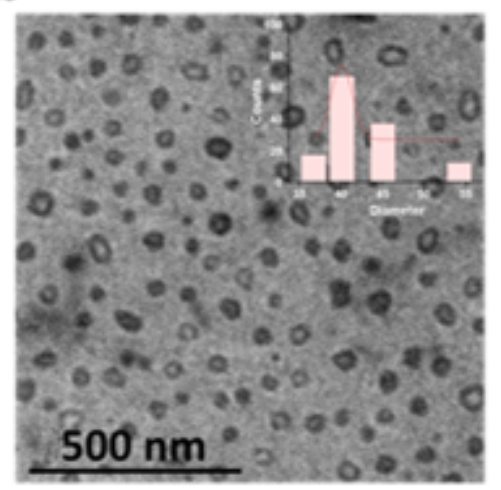

e

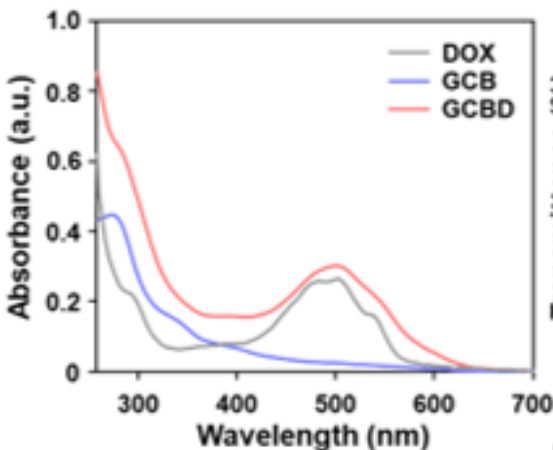

h



C

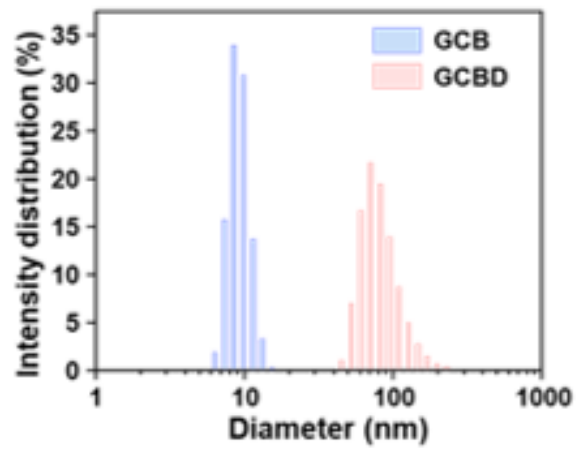

f

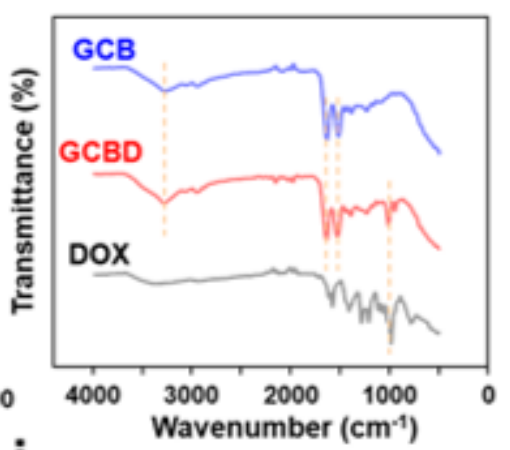

i

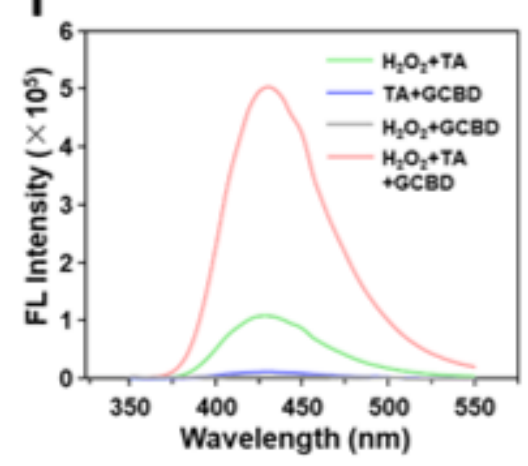




\section{Figure 1}

Characterization of GA-Cu@BSA (GCB NDs) and GA-Cu@BSA-DOX (GCBD NPs). (a) Transmission electron microscope (TEM) image of GCB NDs showing an average size of $8.1 \mathrm{~nm}$. (b) TEM image of GCBD NPs showing an average size of $40.1 \mathrm{~nm}$. (c) Dynamic light scattering (DLS) of GCB NDs and GCBD NPs. (d) The zeta potential of free BSA, free DOX, GCB NDs and GCBD NPs. (e) The absorbance spectra of free DOX, GCB NDs and GCBD NPs. (f) Fourier transform infrared (FT-IR) spectra of free DOX, GCB NDs and GCBD NPs. (g) The release profile of DOX from GCBD NPs at different $\mathrm{pH}$. (h) The release profile of copper ions from GCBD NPs at different $\mathrm{pH}$. (i) The fluorescence spectra of different solutions including TA+ $\mathrm{H}_{2} \mathrm{O}_{2}, T A+G C B D$ NPs, $\mathrm{H}_{2} \mathrm{O}_{2}+$ GCBD NPs and $\mathrm{H}_{2} \mathrm{O}_{2}+\mathrm{TA}+\mathrm{GCBD}$ NPs $(\lambda e x=315 \mathrm{~nm})$.

\section{Figure 2}

The analysis of cell death pathway. (a) Relative cell viability of HUVEC cells after incubation with GCB NDs at different concentrations $(0-200 \mu \mathrm{g} / \mathrm{mL})$ for $24 \mathrm{~h}$. (b) Relative cell viability of $4 \mathrm{~T} 1$ cells after incubation with GCB NDs (0-200 $\mu \mathrm{g} / \mathrm{mL})$, DOX (0-20 $\mu \mathrm{g} / \mathrm{mL})$ or GCBD NPs $(0-20 \mu \mathrm{g} / \mathrm{mL}$ of DOX) for $24 \mathrm{~h}$. (c) Confocal imaging of calcein-AM/PI stained 4T1 cells after incubation with different solutions for $24 \mathrm{~h}$.

(d) The flow cytometry analysis of 4T1 cells after incubation with different solutions for $24 \mathrm{~h}$, showing more significant apoptosis in GCBD NPs-treated cells than other groups $(2.5 \mu \mathrm{g} / \mathrm{mL}$ for DOX). 


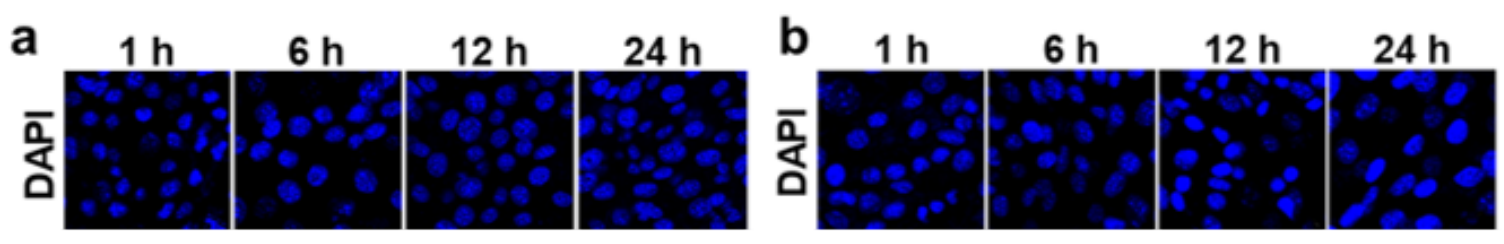

\section{Figure 3}

Cellular uptake of chemotherapy drug. (a-b) Confocal imaging of 4T1 cells demonstrating the cellular uptake of DOX ( $2 \mu \mathrm{g} / \mathrm{mL})$ (a) and GCBD NPs ( $2 \mu \mathrm{g} / \mathrm{mL}$ of DOX) (b) at different time points. The nuclei of cells were stained with DAPI. (c-d) Flow cytometry-measured cellular uptake of free DOX $(2 \mu \mathrm{g} / \mathrm{mL})(\mathrm{c})$ and GCBD NPs $(2 \mu \mathrm{g} / \mathrm{mL}$ of DOX) (d) in $4 \mathrm{~T} 1$ cells at different time points. (e) The semiquantitative 
analysis of cellular uptake corresponding to the confocal images in a\&b. (f) The semiquantitative analysis of cellular uptake corresponding to the flow cytometry results in c\&d.

\section{Figure 4}

In vitro chemo/chemodynamic therapy based on GCBD NPs. (a) Schematic illustration of cellular chemo/chemodynamic therapy mechanism based on GCBD NPs. (b) The GSSG/GSH ratio of GSH solutions (10 mM) after incubation with PBS, GCB NDs or GCBD NPs for $24 \mathrm{~h}$. (c) The relative GSH content in $4 \mathrm{~T} 1$ cells after incubation with different solutions for $24 \mathrm{~h}$. (d) Confocal laser scanning microscopy (CLSM) imaging of 4T1 cells stained with DCFH-DA after various treatments. Scale bar: 20 $\mu \mathrm{m}$.

\section{Figure 5}

The blood circulation and biodistribution of GCBD NPs in mice. (a) The blood circulation of ${ }^{125}$ @GGCBD NPs in Balb-c mice after intravenous (i.v.) injection (20 $\mu$ Ci for ${ }^{125}$, $2.5 \mathrm{mg} \mathrm{kg}^{-1}$ of DOX). (b) The biodistribution of ${ }^{125}$ @GCBD NPs at $4 \mathrm{~h}$ (pink) and $24 \mathrm{~h}$ (gray) after i.v. injection ( $20 \mu \mathrm{Ci}$ for ${ }^{125} \mathrm{l}, 2.5 \mathrm{mg}$ $\mathrm{kg}^{-1}$ of DOX) as determined by measuring the radionuclide intensity in the major organs and tumors. (c) The fluorescence imaging of $4 \mathrm{~T} 1$ tumor-bearing mice at different time points after i.v. administration of Cy5.5-labeled GCB NDs $\left(10 \mathrm{mg} \mathrm{kg}^{-1}\right)$ or GCBD NPs $\left(2.5 \mathrm{mg} \mathrm{kg}^{-1}\right.$ of DOX) (the tumor was highlighted by white dotted circle). The error bars were based on the standard deviation of tree mice in each group.

\section{Figure 6}

Combined chemo/chemodynamic therapy in vivo. (a) Tumor growth curves monitored for 14 days after various treatments. (b) Tumor weight in each group 14 days after each treatment. (c) Hematoxylin and eosin (H\&E) and TUNEL stained tumor slice post specific treatment. The error bars were based on the standard deviation of 6 mice in each group.

\section{Supplementary Files}

This is a list of supplementary files associated with this preprint. Click to download.

- GraphicalAbstract.docx

- SupportingInformation.docx

- Scheme1.png 